Article

\title{
Development and Validation of a UHPLC-qTOF MS Method for the Determination of Sorbitol-Based Nuclear Clarifying Agents in Food Simulants after Migration from Food Contact Materials
}

\author{
Emmanouil D. Tsochatzis ${ }^{1, *(1)}$, Georgios Theodoridis ${ }^{2,3,4}$ (D) and Helen G. Gika ${ }^{2,3,5}$ \\ 1 Department of Food Science, Centre of Innovative Food Research (iFood), Aarhus University, Agro Food Park 48, \\ 8200 Aarhus N, Denmark \\ 2 FoodOmicsGR Research Infrastructure, AUTh Node, Center for Interdisciplinary Research and \\ Innovation (CIRI-AUTH), Balkan Center B1.4, 10th Km Thessaloniki-Thermi Rd, P.O. Box 8318, \\ 57001 Thessaloniki, Greece; gtheodor@chem.auth.gr (G.T.); gkikae@auth.gr (H.G.G.) \\ 3 Biomic AUTh, Center for Interdisciplinary Research and Innovation (CIRI-AUTH), Balkan Center B1.4, \\ 10th Km Thessaloniki-Thermi Rd, P.O. Box 8318, 57001 Thessaloniki, Greece \\ 4 Department of Chemistry, Aristotle University of Thessaloniki, 54124 Thessaloniki, Greece \\ 5 School of Medicine, Aristotle University of Thessaloniki, 54124 Thessaloniki, Greece \\ * Correspondence: Emmanouil.tsochatzis@food.au.dk; Tel.: +45-41893130
}

check for updates

Citation: Tsochatzis, E.D.; Theodoridis, G.; Gika, H.G. Development and Validation of a UHPLC-qTOF MS Method for the Determination of Sorbitol-Based Nuclear Clarifying Agents in Food Simulants after Migration from Food Contact Materials. Appl. Sci. 2021, 11, 3789. https://doi.org/10.3390/app11093789

Academic Editor: Claudio Medana

Received: 8 April 2021

Accepted: 19 April 2021

Published: 22 April 2021

Publisher's Note: MDPI stays neutral with regard to jurisdictional claims in published maps and institutional affiliations.

Copyright: (c) 2021 by the authors. Licensee MDPI, Basel, Switzerland. This article is an open access article distributed under the terms and conditions of the Creative Commons Attribution (CC BY) license (https:// creativecommons.org/licenses/by/ $4.0 /)$.

\begin{abstract}
Nuclear clarifying agents (NCAs) are a class of substances frequently used as additives in the production of polymers to improve their physical properties. Some are EU regulated under Commission Regulation (EU) no. 10/2011 can be used as additives in the production of food contact plastics. However, limited analytical methods for their analysis are currently available, in part due to poor solubility in most common organic solvents and lack of analytical standards of known purity. In this work, a simple and sensitive method was developed to analyze 4 EU-regulated sorbitol-based nucleating agents in food simulants, following solubility studies to establish effective solvents. The method was shown to be accurate and precise and can be used with official food simulant D1 (50\% $v / v$ ethanol $/ \mathrm{H}_{2} \mathrm{O}$ ). Application to other ethanolic simulants is also possible, but due to solubility issues, a posteriori conversion of those simulants into simulant D1 is required. Finally, the method was applied to quantify the target analytes in simulants after migration testing with polypropylene (PP) beverage cups.
\end{abstract}

Keywords: food contact materials; UHPLC-QTOF-MS; nuclear-clarifying agents (NCAs); migration testing; food simulants

\section{Introduction}

Nuclear clarifying agents (NCAs) are a class of additives that have become widely used in the production of several polymers due to their potential to increase optical clarity, physical properties, and resistance to UV radiation, among others. Although important in the production of plastics in several fields of application (e.g., cosmetics and pharmaceutical), but also in food contact materials, these enhanced physical properties are also highly desired in the production of food contact materials (FCMs). That is the case of polypropylene (PP) packaging, in which production NCAs are used to transform that polymer into a more visually attractive alternative than other plastic FCMs like polystyrene (PS) and poly(ethylene terephthalate) (PET) [1,2].

Many NCAs exist in the market, with most of them having chemical structures based on sugar/sugar polyols, such as sorbitol. The most used ones are marketed under different brand names, including Irgaclear ${ }^{\circledR}$, Irgaclear $\mathrm{DM}^{\circledR}$, and Millad ${ }^{\circledR}$. Some NCAs are included in the positive list of additives of the European Commission (EU) Reg. no. 10/2011 for plastic FCMs. That is the case of dibenzylidene sorbitol (DBS, FCM no. 674), bis(methylbenzylidene)sorbitol (FCM no. 752), bis(3,4-dimethylbenzylidene)sorbitol (FCM 
no. 766) and bis(4-propylbenzylidene)propylsorbitol (PBPS, FCM no. 808). Their chemical structures are shown in Figure 1.

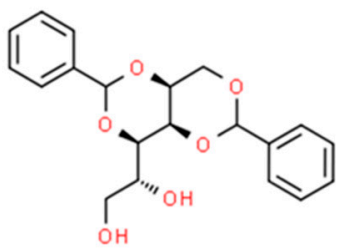

$\underline{\text { FCM 674 }} ;(1,3: 2,4)$ Dibenzylidene sorbitol $\mathrm{M}=358.385$ (DBS)

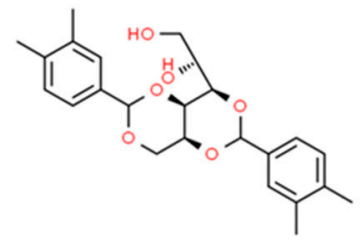

$\underline{F C M 766}$; bis(3,4-dimethylbenzylidene) sorbitol $\mathrm{M}=415.211$ (DMDBS)

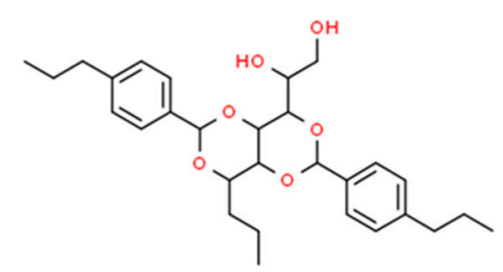

$\underline{F C M 808 ; ~ b i s(4-p r o p y l b e n z y l i d e n e) ~ p r o p y l s o r b i t o l ~}$ $\mathrm{M}=484.624 \mathrm{Da}$ (PBPS)<smiles>Cc1ccc(C2OC3COC(c4ccc(C)cc4)O[C@@H]3C([C@H](O)CO)O2)cc1</smiles>

$\underline{\text { FCM 752; }}$;is(methylbenzylidene)sorbitol $\mathrm{M}=387.180$ (MDBS)

Figure 1. Basic chemical structure of sorbitol-based NCAs highlighted different side chains and their systematic names.

From this group, FCM no. 674, 752 and 766 have no specific migration limits (SMLs), meaning that they can be used without any individual restriction in the production of food contact plastics. FCM no. 808 is the only NCA with an established SML, $5 \mathrm{mg} \mathrm{kg}^{-1}$ food [3].

There has been little research to date on sorbitol-based NCAs in FCMs in the literature [2,4]. The lack of commercial analytical standards with known purity is problematic in analyzing FCM additives [5,6]. That is the case of FCM no. 674, a well-known and highly effective NCA commercialized as the first generation product of his class [2,7-10]. 2,4-bis-O- (benzylidene)sorbitol and 1,3:2,4-bis-O-(p-methylbenzylide- ne)sorbitol, 1,3:2,4bis-O-(3,4-dimethylbenzylidene)sorbitol), using both LC-MS and GC-MS. For the latter, a silylation protocol has been applied to ensure the necessary volatility of the analytes for the GC analysis, but no quantitative results were presented. Although used widely in the production of PP, almost no published analytical data regarding its identification in FCMs can be found in the literature.

Some exceptions include the work of Sternbauer et al., where it was reported an analytical method for determining NCAs in plastic materials using a GC-MS method after microwave-assisted extraction with in situ silyl derivatization. A derivatization step is necessary to analyze these substances with gas chromatography (GC), as they lack the necessary volatility to use that technique. The authors have also reported a recurrent issue with NCAs, which is their poor solubility in most organic solvents. The issue was solved by using tetrahydrofuran (THF) as an extraction solvent [4]. In another work, Lopes et al. reported the identification and analysis of FCM no. 808 migrating from PP beverage cups into food simulant D1 ( $50 \% v / v$ ethanol/water). Found migration levels were within the established EU regulated levels. Almost no commercial analytical standards for NCAs are available at the moment, hindering the retrieval of information at physical and chemical levels [11]. The only work that has presented consistent information on some NCAs mass spectral data is the one by McDonald et al. [2]. However, no analytical method development nor validation and quantification of the analytes were performed.

The present study aimed at developing and validating a precise and rapid UHPLCQTOF-MS method for the analysis of the four NCAs EU regulated as FCM plastic additives. The method was validated in terms of sensitivity, linearity, accuracy and precision, while 
also providing high-resolution mass spectra of the analytes, information that can be used in other future works for their identification in food or food simulants. The method has been applied to migration solutions of different types of commercially available beverage cups. To the best of the author "s knowledge, this work describes the first analytical method for the simultaneous determination of these NCAs.

\section{Materials and Methods}

\subsection{Chemicals}

Ethanol (EtOH), methanol (MeOH), 2-propanol (IsOH), acetone (Acet), acetonitrile $(\mathrm{MeCN}), \mathrm{N}, \mathrm{N}^{\prime}$-dimethylformamide (DMF), N, $\mathrm{N}^{\prime}$-dimethylacetamide (DMA), dimethyl sulfoxide (DMSO), tetrahydrofuran (THF), dichloromethane (DCM) and chloroform $\left(\mathrm{CHCl}_{3}\right)$ were all of CHROMASOLV grade and supplied by Sigma-Aldrich (Steinheim, Germany). LC-MS grade formic acid was supplied by Sigma-Aldrich (Steinheim, Germany). Ultrapure water (18.2 M $\Omega$ ) (Milli-Q, Millipore, Darmstadt, Germany) was used to prepare solutions and food simulants. DBS standard was supplied by TRC Chemicals (Toronto, Canada), bis(methylbenzylidene)sorbitol (MDBS) was supplied by Sigma-Aldrich, while bis(3,4-dimethylbenzylidene)sorbitol (DMDBS) and PBPS were provided by the reference collection of the European Union Reference Laboratory for FCM (EURL-FCM) (Ispra, Italy). No uncertainty estimation was given in the certificate of analysis.

\subsection{Samples}

PP beverage cups were bought at local markets (Denmark). The producers marketed them as being intended mainly for single-use, potentially with alcoholic and/or hot beverages. The volume of two of the cups was $300 \mathrm{~mL}$, with another two with a volume of $200 \mathrm{~mL}$ and $250 \mathrm{~mL}$. The latter was labeled as being made of a PP copolymer (PP-C, heterophasic copolymer). Considering a worst-case scenario, the FCM cups were subjected to article filling migration testing with food simulant D1 at $70{ }^{\circ} \mathrm{C}$ for $2 \mathrm{~h}$, following the recommendations reported for this type of testing [11-14]. This information is summarized in Table 1.

Table 1. Characteristics and experimental testing conditions ${ }^{\mathrm{a}, \mathrm{b}}$ for the PP beverage cups.

\begin{tabular}{cccccccc}
\hline Sample & Material & Type & Intended Use & $\begin{array}{c}\text { Surface Contact } \\
\text { Area }\left(\mathbf{d m}^{2}\right)\end{array}$ & $\begin{array}{c}\text { Volume } \\
(\mathbf{m L})\end{array}$ & $\begin{array}{c}\text { Migration } \\
\text { Experiment }\end{array}$ & $\begin{array}{c}\text { Temperature-Time } \\
\text { Conditions }\end{array}$ \\
\hline PP1 & Polypropylene & Cup & Alcoholic drinks, milk & 4.8 & 300 & Article filling & $70^{\circ} \mathrm{C} \times 2 \mathrm{~h}$ \\
PP2 & $\begin{array}{c}\text { Polypropylene } \\
\text { copolymer }\end{array}$ & Cup & Alcoholic drinks, milk & 3.7 & 250 & Article filling & $70^{\circ} \mathrm{C} \times 2 \mathrm{~h}$ \\
PP3 & $\begin{array}{c}\text { Polypropylene } \\
\text { PP4 }\end{array}$ & Cup & Alcoholic drinks, milk & 4.6 & 300 & Article filling & $70^{\circ} \mathrm{C} \times 2 \mathrm{~h}$ \\
Polypropylene & Cup & Alcoholic drinks, milk & 3.6 & 200 & Article filling & $70^{\circ} \mathrm{C} \times 2 \mathrm{~h}$ \\
\hline
\end{tabular}

a according to Annex III of Commission Regulation (EU) no. 10/2011 [3]. ${ }^{\mathrm{b}}$ According to CEN 13130:2004 for specific migration [15].

\subsection{Solubility Studies}

The solubility of the four NCAs was investigated to select the most appropriate solvents for the preparation of calibration curves and the LC analysis. Experiments were based on the Joint Food and Agriculture Organization of the United Nations (FAO) and World Health Organization (WHO) Expert Committee of Food Additives protocols [16], which also provides solubility classifications for each solubility range (material amount per volume of solvent necessary for its solubilization) $[4,10]$.

Briefly, portions from 2.0 to $50 \mathrm{mg}$ of each NCA were dissolved in different volumes of 11 solvents (MeOH, EtOH, IsOH, Acet, MeCN, DMF, DMA, DMSO, THF, DCM and $\mathrm{CHCl}_{3}$ ). Each sample was vortexed, sonicated and left still for $2 \mathrm{~h}$, according to WHO/FAO protocol [16]. The samples were then inspected both under visible and UV light to determine the presence of insoluble particles. 


\subsection{Calibration and Preparation of Standard Solutions}

Stock solutions containing $1000 \mu \mathrm{g} \mathrm{mL}{ }^{-1}$ of each analyte were prepared gravimetrically using DMF as a solvent and considering the purity of the analytical standards stated by the supplier. Appropriate working solutions were then prepared by dilution with $\mathrm{MeCN} / \mathrm{DMF}$ 1:1 $\mathrm{v} / \mathrm{v}$. Stock solutions were stored at $-20^{\circ} \mathrm{C}$, while the working standards were kept at $4{ }^{\circ} \mathrm{C}$. Fresh working standards were produced every 2 weeks. Both stock and working standard solutions were prepared in amber vials to prevent light-induced degradation or isomeric conversion.

Two multicomponent solutions containing equal concentrations (50 and $10 \mathrm{mg} \mathrm{L}^{-1}$ ) of each compound were prepared on the day of calibration by transferring appropriate volumes of the $1000 \mathrm{mg} \mathrm{L}^{-1}$ stock solutions into $10 \mathrm{~mL}$ volumetric flasks, which were then filled-to-volume with food simulant D1 ( $50 \% v / v$ ethanol in $\left.\mathrm{H}_{2} \mathrm{O}\right)$. A serial dilution of these solutions was followed so to prepare seven multicomponent standard working mixtures of equal concentrations concerning each compound $\left(1,5,10,50,100,500,1000 \mu \mathrm{g} \mathrm{L}^{-1}\right)$. Calibration curves were constructed by plotting the means of triplicate measurements of peak areas against concentrations of the compounds. A blank sample was analyzed prior to the sample analysis (food simulant D1), highlighting that there were no interferences present.

To minimize the contamination of food simulants with plastic phthalates, all glassware was rinsed two times with acetone and hexane and stored in a desiccator over $\mathrm{Al}_{2} \mathrm{O}_{3}$ until further use, as previously reported by the European Commission $[17,18]$.

\subsection{Instrumentation}

\subsubsection{Ultra-High-Performance Liquid Chromatography (UHPLC)}

A UHPLC system (Agilent 1290) was used for chromatographic separations. The analytical column was a Waters BEH C18 $100 \mathrm{~mm} \times 2.1 \mathrm{~mm}, 1.7 \mu \mathrm{m}$ particle size (Waters, USA), temperature-controlled at $40{ }^{\circ} \mathrm{C}$. The mobile phase consisted of $\mathrm{H}_{2} \mathrm{O}$ with $0.1 \%$ formic acid (A) and $\mathrm{MeCN}$ with $0.1 \%$ formic acid (B) at a flow rate of $300 \mu \mathrm{L} \mathrm{min}{ }^{-1}$. The gradient program changed linearly from $50 \%$ to $95 \%$ B in 12 min followed by an isocratic elution for $4 \mathrm{~min}$. An equilibration time of $1 \mathrm{~min}$ was set for the mobile phase to reach initial conditions again. The injection volume was $5 \mu \mathrm{L}$.

\subsection{2. qTOF-MS}

A quadrupole time-of-flight (TOF) mass spectrometer (Agilent 6540 UHD AccurateMass, Agilent, Waldbronn, Germany) was operated in positive ionization mode (Dual ESI probe) with a $4 \mathrm{kV}$ capillary voltage. The source operated at $325^{\circ} \mathrm{C}$, and nitrogen was used as drying gas operated at 40 psi. Furthermore, nitrogen also has been used as nebulizing gas at a flow rate of $10 \mathrm{~L} \mathrm{~min}^{-1}$. The fragmentor, the skimmer and the OCT $1 \mathrm{RF}$ Vpp were operated at $170 \mathrm{~V}, 65 \mathrm{~V}$ and $750 \mathrm{~V}$, respectively, while the TOF-MS acquisition mode set to full scan acquiring data over the $m / z$ range of $100-1600 \mathrm{Da}$, with a 3 spectra/s rate.

The identification of the analytes was performed with the MassHunter software (Agilent, USA). This software provides the most probable formula for the ion under investigation, considering that the molecules are formed with the most common elements selected by the analyst $(\mathrm{C}, \mathrm{H}, \mathrm{O}, \mathrm{N}, \mathrm{Cl}, \mathrm{S}$ and $\mathrm{Na}$ as adduct) with a resolution of 35,000 and MS accuracy $(\Delta \mathrm{m})$ of $0.5 \mathrm{ppm}$. Once the different options for the elemental composition of each accurate mass were known, the molecular structures were searched in Agilent "s Extractable and Leachable Personal Compound Database and Libraries (Agilent Technologies, PCDLs) and in in-house developed databases. Other existing databases, generic chemical databases (i.e., ChemSpider), and using ACD/Labs Spectrus processor and MS Fragmenter (ACD/Labs, Toronto, ON, Canada) were also used. This facilitates the prediction and confirmation, and comparison of fragmentation patterns between experimental with theoretical data. 


\subsection{Method Validation}

Linearity, precision and trueness, limits of detection (LODs) and limits of quantification (LOQs) were assessed and evaluated based on existing international method performance and validation guidelines $[19,20]$. Thus, linearity was assessed with standard mixtures at six levels of concentration for each analyte, while calibration was based on matrix-matched calibration curves in food simulant and evaluated by the linear regression coefficient $\left(\mathrm{R}^{2}\right)$. The method's LODs and LOQs were based on the chromatographic signal-to-noise ratio $(\mathrm{S} / \mathrm{N})$. The mean value and standard deviation of the $\mathrm{S} / \mathrm{N}$ were obtained from 5 chromatograms of the lowest calibration level for each substance. The LOD was calculated as 3 times the $\mathrm{S} / \mathrm{N}$, while the LOQ was calculated as 3 times the LOD $[19,21]$.

Trueness and precision were assessed in food simulant $\mathrm{D} 1(50 \%$ v/v ethanol on $\mathrm{H} 2 \mathrm{O})$, the one with the highest amount of organic solvent and often the most problematic during method validation due to solubility issues [3]. This food simulant was fortified at 3 concentration levels for all the selected analytes based on their linear range. Repeatability was evaluated by performing six replicates of the fortified samples within the same day and by the same operator, while for reproducibility, six replicates of the samples were analyzed on three consecutive days by the same operator. Assessment of analytical method's method trueness was based on calculating the percent relative recovery as the amount found in the fortified sample divided by the known amount added. The three tested concentrations for repeatability and reproducibility were selected based on the method's LOQs and linear range (low, medium, high).

\subsection{Matrix Effect}

Studying the matrix effect (ME) is important when using MS detection. It highlights potential ion suppression/enhancement that can result in interferences during analysis. The ME was evaluated using the following equation $[6,19,22]$ :

$$
\mathbf{M E}=\left(\frac{\mathbf{B}-\mathbf{C}}{\mathbf{C}}\right) \times 100
$$

with B being the slope of regression fit of results and standards added to food simulant D1 after sample preparation, and C the slope of regression fit of results for NCAs standards in food simulant D1. Values from $-10 \%$ up to $10 \%$ indicate no relevant ME, while values higher than $10 \%$ indicate ion enhancement and values lower than $-10 \%$ ion suppression.

\section{Results}

\subsection{Solubility Tests}

Even though the studied NCAs are widely used, analytical studies were carried out to a low extent. One possible explanation may be the very poor solubility of these substances in various commonly used organic solvents, which complicates the preparation of stock solutions and the choice of a proper extraction solvent [2,4].

Therefore, before developing the analytical method, the solubility of the studied NCAs was investigated to select the most appropriate solvent to be used in the studies. The Joint Food and Agriculture Organization of the United Nations (FAO) and World Health Organization (WHO) Expert Committee of Food Additives protocols were followed, as described in Section 2.4 and used by the authors in previous works $[16,23,24]$. The obtained results are provided in Table 2 .

The reported solubility results confirm the few previously reported results, with the NCAs showing very poor solubility in most common organic solvents. Thus, typical solvents, such as methanol, ethanol, acetonitrile or 2-propanol, do not completely dissolve the studied analytes. Moreover, using chlorinated solvents, such as $\mathrm{DCM}$ or $\mathrm{CHCl}_{3}$, does not significantly increase the solubility of the studied NCAs. Hence, more aggressive solvents had to be tested, such as THF, DMA and DMF, to facilitate solubility and subsequently their analysis in the food simulants. As shown in Table 2, the best results were obtained by using DMF and THF. In addition, DMA presented considerable solubility, although slightly 
lower for FCM no. 752 and FCM no. 766. The solubility tests procedures and solubility ranges adapted from $\mathrm{WHO} / \mathrm{FAO}$ protocol [16].

Table 2. Results of solubility studies for the studied NCAs.

\begin{tabular}{|c|c|c|c|c|}
\hline \multirow{2}{*}{ Solvent } & \multicolumn{4}{|c|}{ NCAs } \\
\hline & FCM No. 674 & FCM No. 752 & FCM No. 766 & FCM No. 808 \\
\hline $\mathrm{MeOH}$ & Insoluble & Insoluble & Insoluble & Insoluble \\
\hline EtOH & Very slightly soluble & Very slightly soluble & Very slightly soluble & Very slightly soluble \\
\hline IsOH & Insoluble & Insoluble & Insoluble & Insoluble \\
\hline MeCN & Very slightly soluble & Very slightly soluble & Insoluble & Very slightly soluble \\
\hline Acetone & Insoluble & Insoluble & Insoluble & Insoluble \\
\hline DMSO & Very slightly soluble & Very slightly soluble & Very slightly soluble & Very slightly soluble \\
\hline $\mathrm{CH}_{2} \mathrm{Cl}_{2}$ & Slightly soluble & Insoluble & Very slightly soluble & Insoluble \\
\hline $\mathrm{CHCl}_{3}$ & Slightly soluble & Insoluble & Very slightly soluble & Insoluble \\
\hline DMF & Freely soluble & Freely soluble & Freely soluble & Freely soluble \\
\hline DMA & Freely soluble & Soluble & Soluble & Freely soluble \\
\hline THF & Freely soluble & Freely soluble & Freely soluble & Soluble \\
\hline
\end{tabular}

Note 1: solubility test procedures and solubility ranges adapted from WHO/FAO protocol [16].

These three solvents can be considered quite aggressive and very toxic. Additionally, DMF and DMA are not often selected to be used with UHPLC analysis due to their potential for difficult ionization. In addition, for LC-MS analysis, DMF and especially THF can be considered quite aggressive, where the latter can potentially damage the plastic tubing of an LC system. Therefore, the dilution ratio with $\mathrm{ACN}$ was decided on for developing the method. Despite these considerations, the performed tests showed that using DMF followed by dilutions with ACN lead to good solubility of the analytes, with no occurring effects, problems, or system instability during the UHPLC analysis. However, special attention shall be taken to avoid unwanted effects.

\subsection{Method Validation}

The method was validated in-house, and its LODs, LOQs and upper linear limits in food simulant D1 are presented in Table 3. An illustrative chromatogram of fortified food simulant D1 $\left(50 \% v / v\right.$ ethanol in $\left.\mathrm{H}_{2} \mathrm{O}\right)$ is presented in Figure 2.

All the obtained HR mass spectra for the target analytes are reported as Supplementary Material (Figures S1-S4).

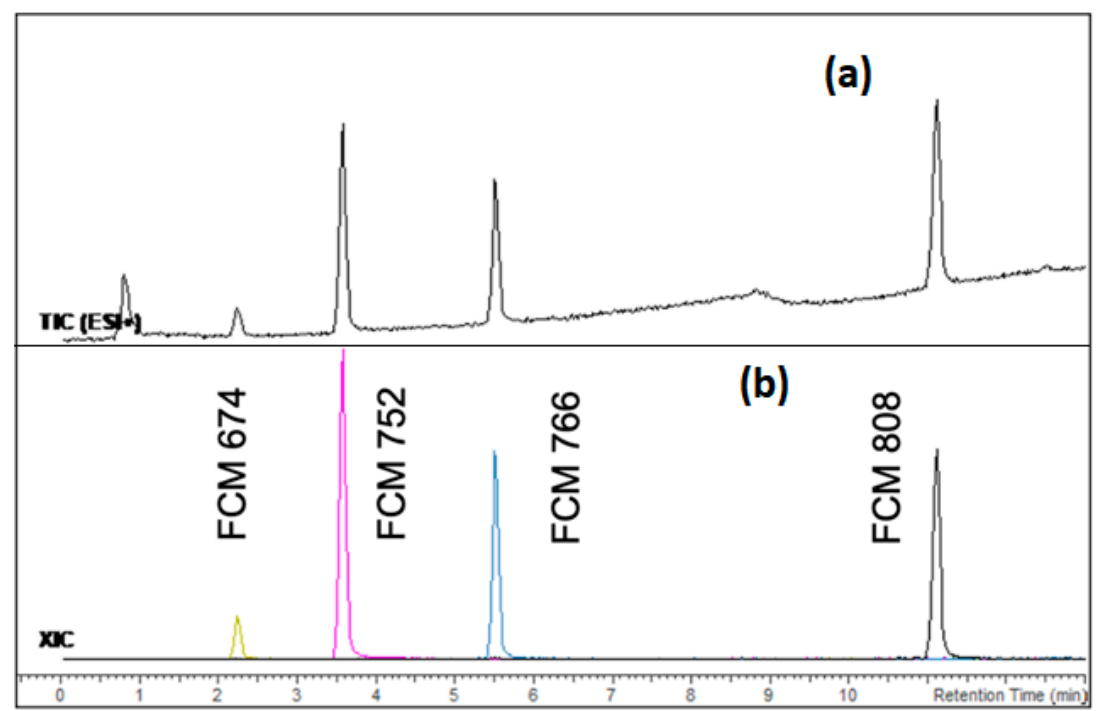

Figure 2. UHPLC-QTOF MS TIC (a) and XIC (b) of the studied target sorbitol-based nucleating agents $\left(100 \mu \mathrm{g} \mathrm{L}^{-1}\right)$. 
Table 3. Analytical features of the validated analytical method.

\begin{tabular}{|c|c|c|c|c|c|c|c|}
\hline Analyte & $\begin{array}{l}\text { Retention Time } \\
\text { (min) }\end{array}$ & $\begin{array}{l}\text { Selected Ion } \\
\quad(\mathrm{m} / \mathrm{z})\end{array}$ & $\begin{array}{c}\text { Linear Regression } \\
\text { Coefficient } \\
\left(\mathbf{R}^{2}\right)\end{array}$ & $\begin{array}{c}\text { LOD } \\
\left.\text { (ng mL } \mathrm{mL}^{-1}\right)\end{array}$ & $\begin{array}{c}\text { LOQ } \\
\left.\text { (ng mL } \mathrm{mL}^{-1}\right)\end{array}$ & $\begin{array}{l}\text { Upper Linear Limit } \\
\quad\left(\mathrm{ng} \mathrm{mL} \mathrm{L}^{-1}\right)\end{array}$ & $\begin{array}{l}\text { Matrix Effect } \\
(\mathrm{ME}, \%)\end{array}$ \\
\hline FCM no. 674 & 2.24 & 359.149 & 0.990 & 4.9 & 16.2 & 500 & 1.2 \\
\hline FCM no. 752 & 3.57 & 387.180 & 0.992 & 1.1 & 3.6 & 500 & -2.1 \\
\hline FCM no. 766 & 5.51 & 415.211 & 0.991 & 1.3 & 4.5 & 500 & -1.1 \\
\hline FCM no. 808 & 11.11 & 485.290 & 0.993 & 2.0 & 6.8 & 500 & 0.9 \\
\hline
\end{tabular}

\subsubsection{Linearity, Sensitivity and Matrix Effect}

The linear regression coefficients $\left(\mathrm{R}^{2}\right)$ were higher than 0.99 for all the studied NCAs, indicating good linearity of the calibration curves [19,21]. LODs and LOQs for the four NCAs in food simulant D1 are given in Table 3. LODs ranged from $1.1 \mathrm{ng} \mathrm{mL}^{-1}$ for FCM no. 752 to $4.9 \mathrm{ng} \mathrm{mL}^{-1}$ for FCM no. 674 , while LOQs ranged from $3.6 \mathrm{ng} \mathrm{mL}^{-1}$ to $16.2 \mathrm{ng} \mathrm{mL}^{-1}$, respectively.

Regarding the ME, results ranged from $-2.1 \%$ to $1.2 \%$, a non-relevant variation that indicates that no signal suppression or enhancement affects the analysis for quantifying the target NCAs in food simulants D1 [19,21].

McDonald et al. reported two analytical methods for analyzing NCAs, namely $(1,3$ : The reported LODs and LOQs for the LC-MS were in line with the ones of the present study [2]. In another work, where derivatization and GC-MS analysis were used, reported LODs and LOQs were higher than the ones of this work $\left(0.05\right.$ to $0.10 \mathrm{mg} \mathrm{L}^{-1}$ and LOQs of 0.17 up to $0.33 \mathrm{mg} \mathrm{L}^{-1}$, respectively) [4]. Additionally, the necessary derivatization steps for GC analysis translates into additional sample preparation steps and increased total analysis time.

\subsubsection{Trueness and Precision}

Results for precision and accuracy (expressed as repeatability and intermediate precision) are given in Table 4.

Table 4. Repeatability and reproducibility results for all the target analytes in food simulant D1 (50\% v/v ethanol in $\left.\mathrm{H}_{2} \mathrm{O}\right)$.

\begin{tabular}{|c|c|c|c|c|c|c|c|c|c|}
\hline \multirow[b]{2}{*}{ Analyte } & \multirow{2}{*}{$\begin{array}{c}\text { Theoretical Added } \\
\text { Amount } \\
\left(\mathrm{ng} \mathrm{mL} \mathrm{mL}^{-1}\right)\end{array}$} & \multicolumn{4}{|c|}{ Repeatability } & \multicolumn{4}{|c|}{ Reproducibility } \\
\hline & & $\begin{array}{c}\text { Experimental } \\
\text { Amount } \\
\left(\mathrm{ng} \mathrm{mL}^{-1}\right)\end{array}$ & SD & $\begin{array}{l}\text { RSD } \\
(\%)\end{array}$ & $\begin{array}{c}\text { Recovery } \\
(\%)\end{array}$ & $\begin{array}{c}\text { Experimental } \\
\text { Amount } \\
\left(\mathrm{ng} \mathrm{mL}^{-1}\right)\end{array}$ & SD & $\begin{array}{l}\text { RSD } \\
(\%)\end{array}$ & $\begin{array}{c}\text { Recovery } \\
(\%)\end{array}$ \\
\hline \multirow{3}{*}{ FCM no. 674} & 20 & 19.4 & 0.4 & 2.2 & 97.2 & 19.9 & 7.0 & 7.2 & 99.5 \\
\hline & 100 & 99.4 & 4.7 & 4.7 & 99.4 & 97.0 & 3.7 & 3.8 & 97.0 \\
\hline & 400 & 357.2 & 30.0 & 8.4 & 89.3 & 372.8 & 9.3 & 11.2 & 93.2 \\
\hline \multirow{3}{*}{ FCM no. 752} & 20 & 19.5 & 0.2 & 1.1 & 97.5 & 19.9 & 3.1 & 3.2 & 99.6 \\
\hline & 100 & 95.2 & 5.2 & 5.5 & 95.2 & 98.7 & 6.0 & 6.4 & 98.7 \\
\hline & 400 & 354.4 & 30.5 & 8.6 & 88.6 & 364.4 & 8.8 & 10.9 & 91.1 \\
\hline \multirow{3}{*}{ FCM no. 766} & 20 & 19.5 & 0.7 & 3.8 & 97.4 & 20.1 & 5.1 & 5.2 & 100.4 \\
\hline & 100 & 96.4 & 6.8 & 7.1 & 96.4 & 98.6 & 5.3 & 5.6 & 98.6 \\
\hline & 400 & 350.0 & 42.7 & 12.2 & 87.5 & 357.6 & 9.2 & 11.8 & 89.4 \\
\hline \multirow{3}{*}{ FCM no. 808} & 20 & 19.4 & 0.4 & 2.2 & 96.8 & 20.2 & 4.8 & 4.9 & 101.2 \\
\hline & 100 & 102.7 & 5.3 & 5.2 & 102.7 & 99.5 & 5.7 & 5.6 & 99.5 \\
\hline & 400 & 364.4 & 31.7 & 8.7 & 91.1 & 354.4 & 9.5 & 11.8 & 88.6 \\
\hline
\end{tabular}

All the results for precision and trueness indicate the good performance of the developed method. Trueness was assessed by performing recovery studies, with values ranging from $87.5 \%$ (FCM no. 766) to $102.7 \%$ (FCM no. 808) for the repeatability and from $88.6 \%$ (FCM no. 808 ) to $101.2 \%$ (FCM no. 808 ) for the intermediate precision. Moreover, pre- 
cision results revealed RSDs ranging from 1.1\% (FCM no. 752) to $12.2 \%$ (FCM no. 766) for repeatability results and from $3.2 \%$ (FCM 752 ) to $11.8 \%$ (FCM 808) for reproducibility [19].

It was, however, noted a general trend of a decrease of recoveries and increase of RSDs (\%) as the concentration level is closer to the upper linear limit (500 $\mathrm{ng} \mathrm{mL}^{-1}$ ). Preliminary tests at higher concentrations using simulant D1 showed a lack of linearity for the studied NCAs, most probably due to solubility issues. This phenomenon can be tentatively explained by the solubility difficulties and particularities of the target NCAs. The $500 \mathrm{ng} \mathrm{mL}^{-1}$ limit was selected to ensure good linearity for the validated method. These solubility difficulties have already been reported by other researchers, which were partially solved by switching to more aggressive organic solvents, such as THF [2,4].

Within this framework, the results for both trueness and precision are certainly within acceptable thresholds $[19,21]$, being between $85-103 \%$ and RSDs lower than $12.5 \%$. These results are comparable to the limited number of relevant analytical and validated methods, like Sternbrauer et al. showing recoveries from $95.8 \%$ up to $104.2 \%$ in real samples for all studied NCAs [4]. Concerning precision, RSDs were lower than $8 \%$, though these results were obtained with a GC-MS method after derivatization of the samples.

\subsubsection{Applicability to Different Food Simulants}

The method was only validated in food simulant D1, as using simulants with less ethanol (simulant $\mathrm{A}$ and $\mathrm{C}$, i.e., $10 \%$ or $20 \% v / v$ ethanol in $\mathrm{H}_{2} \mathrm{O}$, respectively) resulted in the precipitation of the analytes in the chromatographic system and consequent loss of the method "s linearity. For these simulants, a possible solution to overcome the solubility issue is an a posteriori addition of ethanol to convert them into solutions with the same ethanolic content of simulant D1. Preliminary results with analytical standards solutions showed that this is feasible and applicable to spike simulant $A$ and add ethanol to reach the content of simulant D1 and further inject it into the system. This conversion seems to allow the solutions to be analyzed successfully with the developed method. Preliminary tests (not presented) showed comparable results between the transformed simulant A solutions and the "original" simulant D1 solutions, with no statistical differences.

\subsection{Analysis of PP Beverage Cups}

The developed method was used for the analysis of the migrated NCAs from some FCM samples: PP beverage cups. Simulant D1 was used, and the specific migration testing conditions were the ones defined in the EU regulation for this type of item [3]. Results are presented in Table 5.

Table 5. Method application to real FCM samples, after performance of migration testing ${ }^{1}$.

\begin{tabular}{cccccc}
\hline No. & Analyte & \multicolumn{4}{c}{$\mathbf{~ m g ~ k g ~}^{\mathbf{1}}$ of Food $^{\mathbf{2}}$} \\
\hline & & Sample 1 (PP1) & Sample 2 (PP2) & Sample 3 (PP3) & Sample 3 (PP3) \\
\hline 1 & FCM no. 674 & n.d. & 1.1 & n.d. & n.d. \\
2 & FCM no. 752 & n.d. & n.d. & n.d. & n.d. \\
3 & FCM no. 766 & n.d. & n.d. & 1.2 & n.d. \\
4 & FCM no. 808 & 1.9 & n.d. & n.d. & 2.6 \\
\hline
\end{tabular}

${ }^{1}$ migration testing $70{ }^{\circ} \mathrm{C}$ for $2 \mathrm{~h}$ with food simulant D1. ${ }^{2}$ For the transformation of concentrations (ng $\mathrm{mL}^{-1}$ ) to mass fractions of food ( $\mathrm{ng} \mathrm{g}^{-1}$ ), conversion surface-to-volume of $6 \mathrm{dm}^{2} / \mathrm{kg}$ of food was used, according to Commission Regulation (EC) no. 10/2011 [3]; n.d. = below limit of detection (LOD).

The results showed that the migrated mass fractions of the NCAs ranged from $1.1 \mathrm{mg} \mathrm{kg}^{-1}$ up to $2.6 \mathrm{mg} \mathrm{kg}^{-1}$. The samples were compliant with the existing legislative framework [3]. Moreover, it shall be highlighted that no limits are in place for FCM no. 674,752 and 766 . Only FCM no. 808 has an SML of $5 \mathrm{mg} \mathrm{kg}^{-1}$, but $\mathrm{n}$ mean no analytical standard of high purity is available commercially. This additive may originate via hydrolysis of the compound 4-propyl benzaldehyde, an FCM non-intentionally added substance (NIAS) [5]. Neither this substance nor the degradation products of the additives FCM no. 674, no. 752 and no. 766 (benzaldehyde, 3,4-dimethyl benzaldehyde and 4-methyl ben- 
zaldehyde, respectively) were detected in the migration solutions. This seems to indicate that these NCAs are quite resistant in ethanol-water simulants, even at harsh migration conditions $\left(70^{\circ} \mathrm{C} \times 2 \mathrm{~h}\right)$.

\section{Conclusions}

The method presented in this work proved to be a fast, precise and accurate alternative for the quantification of the NCAs regulated at the EU level. The reported analytical method employs direct injection of food simulant D1 immediately after the migration experiments, a simple approach that can be an advantage in laboratory routine analysis working in checking the legislation compliance. The reported low solubility of NCAs in most organic solvents was overpassed in this work by using an aggressive solvent (DMF) for the preparation of calibration solutions and by keeping the concentrations within intervals of known solubility.

The method's LOQs ensure proper quantification at few $\mathrm{ng} \mathrm{mL}^{-1}$ levels. Trueness and precision were in acceptable ranges for all target analytes. The method was successfully applied for the quantification of these substances in different PP monolayer FCM cups after migration testing by article filling. The migration results showed that all the tested cups were compliant with the limits established in EU regulation for the additive FCM no. 808.

Supplementary Materials: The following are available online at https://www.mdpi.com/article/10 .3390/app11093789/s1, Figure S1: HR-QTOF MS of dibenzylidene sorbitol (DBS; FCM 674), Figure S2: HR-QTOF MS of bis(methylbenzylidene)sorbitol (DMBS; FCM 752), Figure S3: HR-QTOF MS of bis(4-propylbenzylidene) propyl sorbitol (PBPS; FCM 808), Figure S4: HR-QTOF MS of bis(3,4dimethylbenzylidene) sorbitol (DMBS; FCM 766).

Author Contributions: Conceptualization, E.D.T. and G.T.; methodology, E.D.T.; software, E.D.T.; validation, E.D.T., H.G.G. and G.T.; formal analysis, E.D.T.; investigation, E.D.T.; resources, E.D.T. and G.T.; data curation, E.D.T. and H.G.G.; writing—original draft preparation, E.D.T..; writing-review and editing, E.D.T., H.G.G. and G.T.; visualization, E.D.T.; supervision, E.D.T. and G.T.; project administration, E.D.T. All authors have read and agreed to the published version of the manuscript.

Funding: This research received no external funding.

Institutional Review Board Statement: Not applicable.

Informed Consent Statement: Not applicable.

Acknowledgments: The authors would like to thank Joao Alberto Lopes from the European Commission (Joint Research Centre, Directorate F-Health, Consumers and Reference Materials), for his help and support for the realization of this work. The authors would like to acknowledge that this work was supported by the framework of the European Cooperation in Science and Technology (COST) "Circul-a-bility" action.

Conflicts of Interest: The authors declare no conflict of interest.

\section{References}

1. Alberto Lopes, J.F.; Tsochatzis, E.D.; Emons, H.; Hoekstra, E. Development and Validation of an HPLC Method with Fluorescence Detection for the Determination of Fluorescent Whitening Agents Migrating from Plastic Beverage Cups. Food Addit. Contam. Part A 2018, 35, 1438-1446. [CrossRef] [PubMed]

2. McDonald, J.G.; Cummins, C.L.; Barkley, R.M.; Thompson, B.M.; Lincoln, H.A. Identification and Quantitation of Sorbitol-Based Nuclear Clarifying Agents Extracted from Common Laboratory and Consumer Plasticware Made of Polypropylene. Anal. Chem. 2008, 80, 5532-5541. [CrossRef] [PubMed]

3. European Commission Commission Regulation (EU). No 10/2011. Off. J. Eur. Union 2011. No 10/2011. [CrossRef]

4. Sternbauer, L.; Dieplinger, J.; Buchberger, W.; Marosits, E. Determination of Nucleating Agents in Plastic Materials by GC/MS after Microwave-Assisted Extraction with in Situ Microwave-Assisted Derivatization. Talanta 2014, 128, 63-68. [CrossRef]

5. Nerin, C. The Challenge of Identifying Non-Intentionally Added Substances from Food Packaging Materials: A Review. Anal. Chim. Acta 2013, 775, 14-24. [CrossRef]

6. Tsochatzis, E.D.; Alberto Lopes, J.; Kappenstein, O.; Tietz, T.; Hoekstra, E.J. Quantification of PET Cyclic and Linear Oligomers in Teabags by a Validated LC-MS Method-In Silico Toxicity Assessment and Consumer's Exposure. Food Chem. $2020,317,126427$. [CrossRef] 
7. Barczewski, M.; Dobrzyńska-Mizera, M.; Dudziec, B.; Sterzyński, T. Influence of a Sorbitol-Based Nucleating Agent Modified with Silsesquioxanes on the Non-Isothermal Crystallization of Isotactic Polypropylene. J. Appl. Polym. Sci. 2014, $131,40131$. [CrossRef]

8. Dobrzyńska-Mizera, M.; Dutkiewicz, M.; Sterzyński, T.; Di Lorenzo, M.L. Polypropylene-Based Composites Containing SorbitolBased Nucleating Agent and Siloxane-Silsesquioxane Resin. J. Appl. Polym. Sci. 2016, 133, 43476. [CrossRef]

9. Katsuno, S.; Yoshinaga, M.; Kitade, S.; Sanada, Y.; Akiba, I.; Sakurai, K.; Masunaga, H. Crystallization Kinetics of Polypropylene Containing a Sorbitol Nucleating Agent. Polym. J. 2013, 45, 87-93. [CrossRef]

10. Nagarajan, K.; Levon, K.; Myerson, A.S. Nucleating Agents in Polypropylene. J. Therm. Anal. Calorim. 2000, 59, 497-508. [CrossRef]

11. Alberto Lopes, J.; Tsochatzis, E.D.; Robouch, P.; Hoekstra, E. Influence of Pre-Heating of Food Contact Polypropylene Cups on Its Physical Structure and on the Migration of Additives. Food Packag. Shelf Life 2019, 20, 100305. [CrossRef] [PubMed]

12. Dehouck, P.; Tsochatzis, E.; Lopes, J.A.; Cizek-Stroh, A.; Robouch, P.; Hoekstra, E. Determination of the Mass Fractions of PBT and PET Oligomers in Food Simulant D; Joint Research Centre: Ispra, Italy, 2018; p. 52.

13. Tsochatzis, E.; Dehouck, P.; Lopes, J.A.; Emteborg, H.; Robouch, P.; Hoekstra, E. Determination of PBT Cyclic Oligomers in and Migrated from Food Contact Materials; Joint Research Centre Publication Office of the European Commission: Ispra, Italy, $2019 ;$ p. 60.

14. Tsochatzis, E.D.; Alberto Lopes, J.; Dehouck, P.; Robouch, P.; Hoekstra, E. Proficiency Test on the Determination of Polyethylene and Polybutylene Terephthalate Cyclic Oligomers in a Food Simulant. Food Packag. Shelf Life 2020, 23, 100441. [CrossRef] [PubMed]

15. Comite Europeen de Normalisation CEN. 13130:2004, Materials and Articles in Contact with Foodstuffs; Part 1: Guide to Test Methods for the Specific Migration of Substances from Plastics to Food and Food Simulants and the Determination of Substances in Plastics and the Selection of Conditions of Exposure to Food Simulants; Comite Europeen de Normalisation: Brussels, Belgium, 2004.

16. Joint FAO/WHO Expert Committee of Food Additives. Joint FAO/WHO Expert Committee of Food Additives. Combined Compendium of Food Additives Specifications: Analytical Methods, Test Procedures and Laboratory Solutions Used by and Referenced in the Food Additive Specifications; FAO: Rome, Italy, 2006.

17. Tsochatzis, E.D.; Alberto Lopes, J.; Hoekstra, E.; Emons, H. Development and Validation of a Multi-Analyte GC-MS Method for the Determination of 84 Substances from Plastic Food Contact Materials. Anal. Bioanal. Chem. 2020, 412, 5419-5434. [CrossRef] [PubMed]

18. European Commission. Joint Research Centre Standard Operating Procedure for the Determination of Phthalates in Wine and Spirits; In-House Validated by the EC-JRC-IRMM; Institute for Reference Materials and Measurements: Geel, Belgium, 2013.

19. European Commission. Guidance Document on Analytical Quality Control and Method Validation Procedures for Pesticide Residues and Analysis in Food and Feed. SANTE/11813/2017; Directorate-General for Health and Food Safety: Brussels, Belgium, 2017 ; Volume 46.

20. Tsochatzis, E.D.; Gika, H.; Theodoridis, G. Development and Validation of a Fast Gas Chromatography Mass Spectrometry Method for the Quantification of Selected Non-Intentionally Added Substances and Polystyrene/Polyurethane Oligomers in Liquid Food Simulants. Anal. Chim. Acta 2020, 1130, 49-59. [CrossRef] [PubMed]

21. Thompson, M.; Ellison, S.L.R.; Wood, R. Harmonized Guidelines for Single-Laboratory Validation of Methods of Analysis (IUPAC Technical Report). Pure Appl. Chem. 2002, 74, 835-855. [CrossRef]

22. Tsochatzis, E.D.; Begou, O.; Gika, H.G.; Karayannakidis, P.D.; Kalogiannis, S. A Hydrophilic Interaction Chromatography-Tandem Mass Spectrometry Method for Amino Acid Profiling in Mussels. J. Chromatogr. B 2017, 1047, 197-206. [CrossRef] [PubMed]

23. Lopes, J.F.A.; Simoneau, C. Solubility of Polyvinyl Alcohol in Ethanol. EFSA Supporting Publ. 2014, 11. [CrossRef]

24. Tsochatzis, E.D.; Lopes, J.A.; Holland, M.V.; Reniero, F.; Emons, H.; Guillou, C. Isolation, Characterization and Structural Elucidation of Polybutylene Terephthalate Cyclic Oligomers and Purity Assessment Using a 1H QNMR Method. Polymers 2019, 11, 464. [CrossRef] [PubMed] 